\title{
Desmin expression in rhabdomyosarcoma: influence of the desmin clone and immunohistochemical method
}

\author{
L Pollock, D Rampling, S E Greenwald, M Malone
}

\begin{abstract}
Aim-To determine which, if any, of five commercially available desmin clones is most reliable at labelling desmin filaments and whether the enhanced polymer one step (EPOS) method of labelling is of any advantage in the routine diagnostic laboratory.

Methods-Thirty four rhabdomyosarcomas from the files at The Hospital for Sick Children, Great Ormond Street, London, were studied. Four different desmin clones, DE-R-11, D33, DE-U-10, and PDE, were applied to each using the conventional extravidin biotin peroxidase method. The D33 clone was also applied using the EPOS method.

Results-The EPOS method incorporating D33 persistently scored more cells as desmin positive and was positive in four cases which were negative on staining with the other clones.

Conclusions-The D33 desmin clone used with the EPOS method is more reliable for identifying desmin filaments in tumours than other desmin antibodies tested. Different desmin clones using a routine technique label different rhabdomyosarcoma cells and therefore it is justifiable to use more than one clone.

(f Clin Pathol 1995;48:535-538)
\end{abstract}

Keywords: Desmin, rhabdomyosarcoma, immunohistochemistry.

Tumours of skeletal and smooth muscle may pose difficulties in diagnosis. Desmin is a protein found in cells of muscle origin ${ }^{1}$ and desmin antibodies are now well established as the best routine marker for identifying rhabdomyosarcomas. ${ }^{23}$ In evaluating the effectiveness of the new enhanced polymer one step (EPOS) immunohistochemical method in a rhabdomyosarcoma case, we noted that the number of positively labelled cells and the intensity of staining varied enormously between the EPOS method and the usual extravidin biotin peroxidase technique. We decided to investigate this phenomenon further by comparing the effectiveness of different desmin clones and the two different methods of applying the antibody.

Desmin is one of the family of intermediate filaments composed of a non-helical headpiece, an $\alpha$ helical middle domain and a $C$ terminal tailpiece. ${ }^{4}$ Antibodies have been raised against different portions of the protein, each being identified by its clone code-for example, DER 11 and D33. ${ }^{5}$ An ever increasing array of different antibody clones is available commercially for routine immunohistochemical use. $^{6}$

The EPOS technique is a new method of immunohistochemical labelling. It is a direct staining technique made possible by the linking of the primary antibody to horseradish peroxidase (HRP) by a polymer which then complexes with a greater number of enzyme molecules than the antibody alone, thus affecting amplification without the need for multilayering. The striking advantage of the EPOS system is in time saved and the fewer steps required. There is no need for titration, reducing the risk of errors, and a result can be obtained within a few hours.

To evaluate which desmin clone and which labelling method would be most useful in our laboratory, we compared four different desmin clones and with one clone we used the EPOS method as well as the routine extravidin biotin technique. As parts of the desmin molecule share extensive homology with other intermediate filaments, we also labelled 50 cases of non-rhabdomyosarcoma childhood tumours with the same desmin clones to assess the possibility of cross-reactivity.

\section{Methods}

Thirty four cases of rhabdomyosarcoma (26 embryonal and seven alveolar), confirmed by electron microscopy, and 10 cases each of neuroblastoma, lymphoma, Wilm's tumour, primitive neuroectodermal tumour (PNET), and retinoblastoma were retrieved from the files of Great Ormond Street Hospital for Sick Children, London. All of the tumours had been fixed in formalin and embedded in paraffin wax. Sections $(3 \mu \mathrm{m})$ were cut, dewaxed and rehydrated. The slides were labelled with four different desmin clones using a standard extravidin biotin peroxidase technique. Endogenous peroxidase was blocked in all slides by incubating with $10 \%$ hydrogen peroxide for 20 minutes. Details of digestion, incubation times and antibody source are given in table 1 . Slides were then incubated with the appropriate second antibody. For the mouse monoclonal, biotinylated goat antimouse at a 1 in 50 dilution for one hour and for the rabbit polyclonal, biotinylated goat antirabbit at a 1 in 200 dilution for 30 minutes.

The slides were washed in phosphate buffered saline (PBS) and incubated with either 


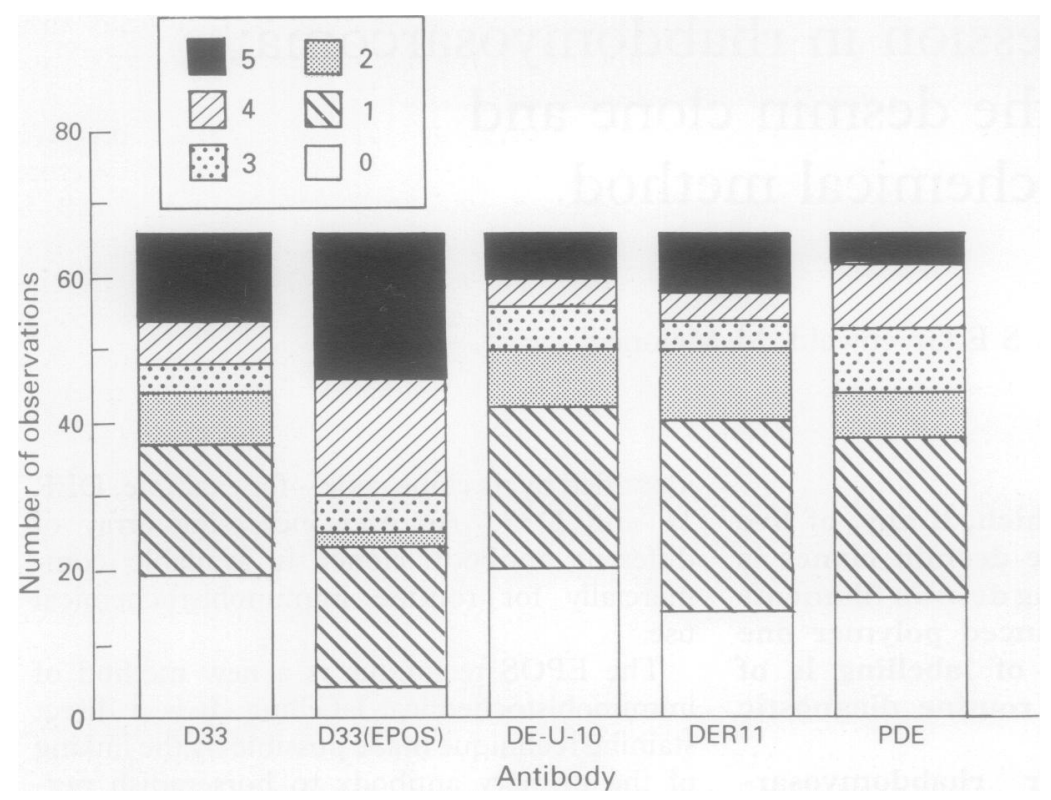

Figure 1 Number of cases which fell into each of the five different percentage labelling groups for each of the 66 observations per antibody: $0=0 \% ; 1=1-5 \% ; 2=6-25 \% ; 3=$ $26-45 \% ; 4=46-65 \% ; 5=>65 \%$

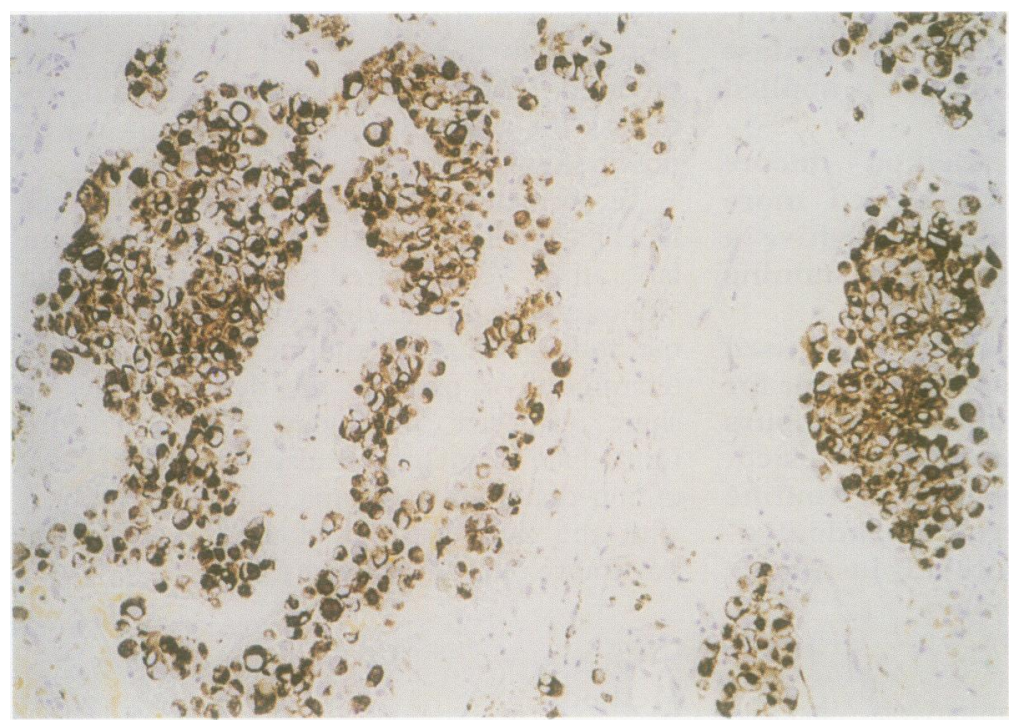

Figure 2 D33 EPOS labelling of the same field in the same rhabdomyosarcoma case as in fig 3 to compare the number of tumour cells labelled.

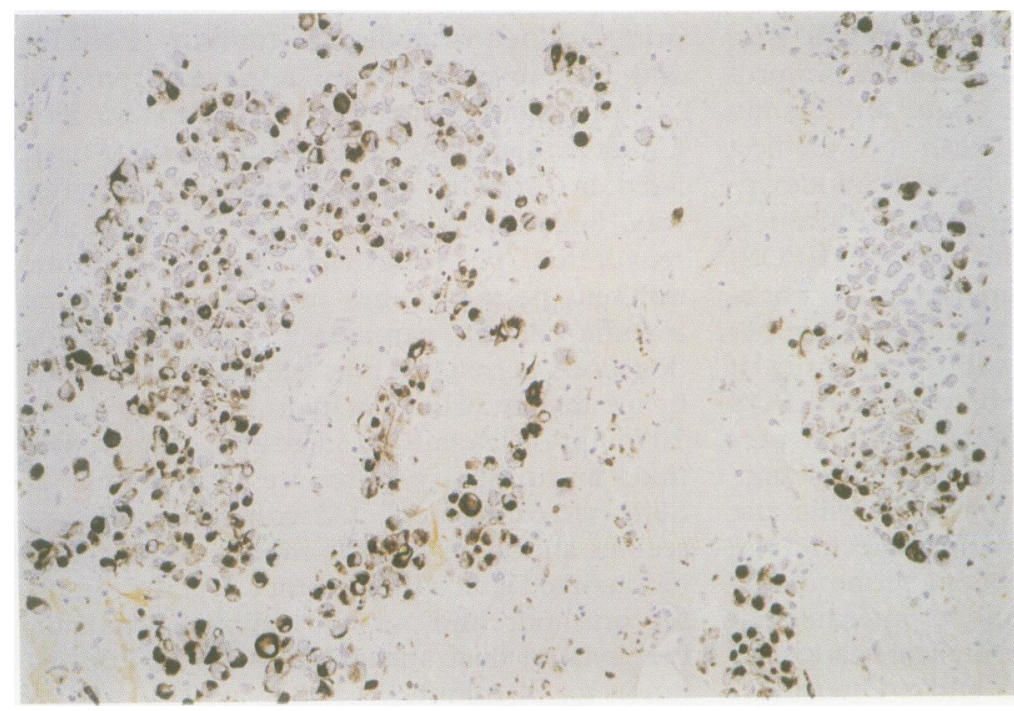

Figure 3 The same field as shown in fig 2 labelled with desmin clone DE-U-10. Extravidin biotin peroxidase method.
Table 1 Digestion, dilution and source of the four different antibodies

\begin{tabular}{lllll}
\hline Antibody & Type & Protease & Dilution & Source \\
\hline DER11 & $\mathrm{mm}$ & $5 \mathrm{mins}$ & 1 in 75 & Dako \\
D33 & $\mathrm{mm}$ & none & 1 in 50 & Dako \\
DE-U-10 & $\mathrm{mm}$ & 5 mins & 1 in 150 & Sigma \\
PDE & $\mathrm{rp}$ & none & 1 in 50 & Europath \\
\hline
\end{tabular}

Dako, Glostrup, Denmark; Sigma, Poole, Dorset, UK; Europath, Cornwall, UK.

$\mathrm{mm}=$ mouse monoclonal; $\mathrm{rp}=$ rabbit polyclonal

mouse monoclonal antiserum (diluted 1 in 50) for one hour or for the PDE clone, rabbit polyclonal antiserum (diluted 1 in 200) for 30 minutes. The peroxidase activity was developed with 3'3-diaminobenzidine (DAB) for 10 minutes. All incubations were carried out at room temperature. The slides were then counterstained with Mayer's haematoxylin. Negative controls omitted the primary antibody; appropriate positive controls were run in parallel.

For the D33 EPOS method, the slides were dewaxed and rehydrated and then incubated for 20 minutes with $100 \%$ hydrogen peroxide in distilled water, rinsed and placed in PBS for five minutes. The slides were then incubated at room temperature with EPOS antidesmin/ HRP (Dako, Glostrup, Denmark) for 60 minutes, rinsed in PBS for five minutes and incubated with DAB for 10 minutes.

All of the slides were examined by two independent observers and coded such that the observers did not know which antibody had been applied. The slides were scored according to percentage of tumour cells labelled.

\section{Results}

The rhabdomyosarcoma cases were scored by each independent observer according to the number of cells labelled. Intensity of labelling was not scored separately as there was no quantitative relevance in this study. A cell was scored if it was clearly positively labelled regardless of depth of colour. To quantify the percentage of cells labelled, the slides were grouped and a score applied to each group as shown in table 2 .

The scores were then added for each clone and plotted onto a bar chart (fig 1). Using the $\chi^{2}$ test, there was no statistically significant interobserver error and so the two results could be added together. One case had no tumour left in the block.

Two cases which behaved as rhabdomyosarcoma clinically and which had features of rhabdomyosarcoma on electron microscopy were negative on staining with all desmin clones by both methods; one was an alveolar rhabdomyosarcoma and the other embryonal.

The EPOS D33 label produced a positive result in four cases when all the other antibodies were negative. Two antibodies, DE-U-10 and D33 (routine method) were negative when DER11, PDE and D33 were positive on EPOS in four and two cases, respectively.

The cumulative results per desmin clone for the rhabdomyosarcoma cases ( 33 cases each 
Table 2 Scoring for each percentage group

\begin{tabular}{ll}
\hline Per cent labelled & Score \\
\hline 0 & 0 \\
$1-5$ & 1 \\
$6-25$ & 2 \\
$26-45$ & 3 \\
$46-65$ & 4 \\
$>65$ & 5 \\
\hline
\end{tabular}

with a result from two observers making 66 observations in total) ( $y$ axis) are shown in fig 1. D33 EPOS had the lowest score for no labelling at all in a case-that is, the white area at the base of the chart. Between the other four antibodies, although DE-U-10 is marginally higher for no positive labelling, the difference is not significant ( $\chi^{2}$ test). Similarly, D33 EPOS has the highest number of high scoring observations (dark block at the top of the chart) and if the two top blocks are considered-that is, any slide with more than $46 \%$ labelled cells, D33 EPOS picks up twice as many slides as the next highest scorer which is D33 using the routine method. Figure 2 shows one rhabdomyosarcoma case labelled by D33 EPOS and fig 3 shows the same field labelled by DEU-10. The comparison clearly demonstrates that far more rhabdomyosarcoma cells are labelled with the D33 EPOS method.

None of the 50 cases of childhood nonrhabdomyosarcoma tumours were positive on staining with any of the desmin clones. None of the 10 Wilm's tumours showed rhabdomyoblastic differentiation.

\section{Discussion}

Rhabdomyosarcoma is a rare tumour but accounts for $5 \%$ of all paediatric malignant tumours. ${ }^{7}$ Distinguishing rhabdomyosarcoma from other childhood small round cell tumours-for example, lymphoma, neuroblastoma and PNET, may be difficult but is important as the treatment protocols and prognoses vary between the different diseases. ${ }^{8}$

Age, site, clinical presentation, and imaging contribute to the diagnosis but histological confirmation is necessary. The development of desmin antibodies has made a significant contribution to easing this diagnostic process but they are not foolproof. ${ }^{9-11}$

Desmin is a member of the intermediate filament family of proteins and was initially thought to be specific to skeletal, cardiac and smooth muscle. More recently, this has been shown not to be absolute as myofibroblasts may also express desmin. ${ }^{12}$ Desmin antibodies became available for use as immunohistochemical markers in the early 1980s and since then have become well established as an aid in the diagnosis of tumours of muscle origin. ${ }^{13}$ Desmin antibodies have proliferated such that there are now a large number of commercially available clones. Although the sensitivity and specificity of desmin as a marker of rhabdomyosarcoma remains high, other tumours can also be labelled with desmin antibodies, notably glial tumours ${ }^{14}$ and, of more relevance to paediatric tumours, there is one report of positive staining with desmin antibodies in childhood neuroectodermal tumours. $^{15}$

Most biopsy specimens of small round cell tumours of childhood are small and therefore the antibody which reliably labels most cells is the most valuable for diagnosis. From the results of our study, although there was no significant difference in the number of positively labelled cases with the routine extravidin biotin peroxidase technique between the different antibodies, there was a difference in the groups of cells that the different desmin clones labelled-that is, two different clones may both be positive but due to labelling of different rhabdomyosarcoma cells. This means that in a small biopsy specimen there is a greater chance of identifying desmin positive cells if two different clones are used.

The most striking difference in labelling was that between the routine method and the EPOS method. Four of the cases which were negative on staining with all antibodies by the routine method were positive with D33 EPOS, an important finding as a desmin negative result may suggest a diagnosis other than rhabdomyosarcoma.

As fig 1 clearly shows, D33 EPOS had the least number of negatively labelled cases and the greatest number of positively labelled cases. This difference was sustained not only against the different desmin clones but also against the same D33 clone using the routine labelling method. Therefore, the clone is not more sensitive but the technique permits greater antigen retrieval. To test the hypothesis that D33 EPOS is significantly better at labelling desmin than the other groups, we applied the $\chi^{2}$ test which gave $\mathrm{p}<0.001$.

DE-U-10 and D33 were the only two antibodies that produced negative results, in four and two cases, respectively, when all of the others were positive. However, D33 did have the second highest score for $46 \%$ or more positively labelled cells.

The two main advantages of the EPOS method with desmin antibodies are (1) in a small biopsy sample there is a greater chance of picking up positive cells and (2) the result is obtained much faster. The routine method takes about four to five hours from the time appropriately dried sections are available, whereas the EPOS method takes about one and a half hours. The latter method is also much simpler, titrations are not required and so can be performed by someone with less specialised training. The time saving and the saving of highly qualified staff offsets the extra expense of the EPOS kits, which cost approximately $£ 2.00$ per slide.

We conclude that with the routine method desmin clones labelled the same overall number of rhabdomyosarcoma cases but the cases labelled were different and therefore for the greatest chance of accurately assessing the muscle of origin of a tumour, more than one clone should be used. The EPOS method for detecting desmin is fast and accurate with significantly more cells being labelled than with the routine extravidin biotin technique. If only 
one desmin clone is to be used with the routine method, we found D33 to be the best.

1 Small JV, Sobieszek A. Studies on the function and composition of the $10 \mathrm{~nm}(100 \mathrm{~A})$ filaments of vertebrate smooth muscle. F Cell Sci 1977;23:243-68.

2 Atlmannsberger M, Weber K, Droste R, Osborn M. Desmin is a specific marker for rhabdomyosarcoma of human and rat origin. Am 7 Pathol 1985;118:85-95.

3 Dias P, Kumar P, Marsden HB, Morris-Jones PH, Birch J, Swindell R, et al. Evaluation of desmin as a diagnostic Swindell R, et al. Evaluation of desmin as a diagnostic and prognostic marker of childhood rhabdomyosarcoma

$4 \mathrm{Ip} \mathrm{W,} \mathrm{Heuser} \mathrm{JE.} \mathrm{Subunit} \mathrm{structure} \mathrm{of} \mathrm{desmin} \mathrm{and} \mathrm{vimentin}$ protofilaments and how they assemble into intermediate filaments. Ann N Y Acad Sci 1985;455:185-99.

5 Debus E, Weber K, Osborn M. Monoclonal antibodies to desmin, the muscle-specific intermediate filament protein. EMBO F 1983;2:2305-12.

6 Wick MR. Antibodies to desmin in diagnostic pathology. In: Wick MR, Siegal GP, eds. Monoclonal antibodies in diagnostic immunohistochemistry. New York: Marcel Dekker Inc., 1988:93-114

7 Carter RL, Jameson CF, Philp ER, Pinkerton CR. Comparative phenotypes in rhabdomyosarcoma and developing skeletal muscle. Histopathology 1990;17:301-9.
8 Malone M. Soft tissue tumours in childhood. Histopathology 1993;23:203-16.

9 Miettenen M, Lehto V-P, Badley RA, Virtanen I. Alveolar rhabdomyosarcoma: demonstration of the muscle type of intermediate filament, desmin, as a diagnostic aid. $A m \mathcal{F}$ Pathol 1982;108:246-51.

10 Tsokos $M$. The role of immunocytochemistry in the diagnosis of rhabdomyosarcoma. Arch Pathol Lab Med 1986; 110:776-8.

11 Parham DM, Webber B, Holt H, Williams WK, Maurer H. Immunohistochemical study of childhood rhabdomyosarcomas and related neoplasms. Results of an Intergroup Rhabdomyosarcoma study project. Cancer Intergroup Rhab

12 Skalli O, Schurch W, Seemayer T, Lagace R, Montandon $\mathrm{D}$, Pittet B, et al. Myofibroblasts from diverse pathological settings are heterogeneous in their content of actin isoforms and intermediate filament proteins. Lab Invest 1989; 60:275-85.

3 Ordonex NG. Antidesmin antibodies. Am 7 Clin Pathol 1990;93:430-1

14 Truong LD, Rangdaeng S, Cagle P, Ro JY, Hawkins H, Font RL. The diagnostic utility of desmin: a study of 584 cases and review of the literature. Am f Clin Pathol 1990; 93:305-14.

15 Parham DM, Dias P, Kelly DR, Rutledge JC, Houghton P. Desmin positivity in primitive neuroectodermal tumours of childhood. Am $\mathcal{F}$ Surg Pathol 1992;16:483-92. 